\title{
BMJ Open Platelet transfusion for neonates with thrombocytopaenia: protocol for a systematic review
}

\author{
Dengjun Liu, ${ }^{1,2}$ Jinlin Wu, ${ }^{1,2}$ Tao Xiong (D) , ${ }^{1,2}$ Yan Yue, ${ }^{1,2}$ Jun Tang (1D 1,2
}

To cite: Liu D, Wu J,

Xiong T, et al. Platelet

transfusion for neonates with

thrombocytopaenia: protocol for a systematic review. BMJ Open 2020;10:e039132. doi:10.1136/ bmjopen-2020-039132

\section{- Prepublication history and} additional material for this paper are available online. To view these files, please visit the journal online (http://dx.doi. org/10.1136/bmjopen-2020039132).

Received 05 April 2020 Revised 20 September 2020 Accepted 29 September 2020

Check for updates

(C) Author(s) (or their employer(s)) 2020. Re-use permitted under CC BY-NC. No commercial re-use. See rights and permissions. Published by BMJ.

'Department of Pediatrics, West China Second University Hospital, Sichuan University, Chengdu, China

${ }^{2}$ Key Laboratory of Birth Defects and Related Diseases of Women and Children (Sichuan University) Ministry of Education, Sichuan University, Chengdu, China

Correspondence to

Dr Tao Xiong;

tao_xiong@126.com

\section{ABSTRACT}

Introduction Thrombocytopaenia is one of the most common haemostatic abnormalities among neonates. It affects approximately one-quarter of neonates admitted into neonatal intensive care units and may lead to a high risk of bleeding and mortality, which are substantial causes for concern by neonatologists. Platelet transfusion (PT) is a specific treatment for thrombocytopaenia. To date, PT thresholds are diverse since the associations between low platelet count and negative outcomes are not clear. We propose this protocol for a systematic review to collect and assess evidence concerning the best PT threshold to reduce mortality, bleeding and major morbidity among neonates with thrombocytopaenia.

Methods and analysis The systematic review will be performed according to the Cochrane Handbook for Systematic Review of Interventions, the Preferred Reporting Items for Systematic Reviews and MetaAnalyses statement, and the Grading of Recommendations Assessment, Development and Evaluation system. Two independent researchers will perform the study selection, data extraction/coding, quality assessment and further analyses of the included studies, with disagreements being resolved by a third researcher. A systematic search of the literature will be conducted in the PubMed, Cochrane Library and Embase databases from database inception through 13 October 2020. All randomised controlled trials, cohort studies and case-control studies will be included without any restrictions regarding publication date or language. The primary outcomes will comprise in-hospital mortality and bleeding episodes. Endnote X9 and Review Manager V.5.3 software will be used to manage the selection process and statistical analysis, respectively. If the included studies are sufficient and homogeneous for any of the outcomes, a quantitative synthesis (metaanalysis) may be performed. Otherwise, we will conduct a narrative systematic review of the results.

Ethics and dissemination Ethical approval is not required for this study because the data will be obtained from published studies and will not include individual patient data. The results of this study are anticipated to be published in a peer-reviewed journal.

PROSPERO registration number CRD42020169262.

\section{BACKGROUND}

Thrombocytopaenia, defined as a platelet count less than $150 \times 10^{9} / \mathrm{L}$, is a common haemostatic abnormality among neonates,

\section{Strengths and limitations of this study}

This study will be the most recent systematic review to evaluate the platelet transfusion threshold for neonates with thrombocytopaenia based on recent evidence. We will include randomised controlled trials and observational studies and separately combine the results of each study design.

- Comprehensive and extensive analyses of the outcomes, including in-hospital mortality, bleeding events, morbidity, adverse effects of transfusion and length of stay, will be performed.

- The review will be performed according to the Cochrane Handbook and the Preferred Reporting Items for Systematic Reviews and Meta-Analyses statement.

- Formal risk of bias analyses will be performed. The quality of evidence will be affected by the bias in original studies.

The results of this systematic review may be helpful for both clinical decisions and further study.

particularly premature infants. ${ }^{12}$ The aetiology of thrombocytopaenia is complicated and involves multiple factors, including abnormal immunity, infection and asphyxia. ${ }^{3-7}$ Thrombocytopaenia may be a sole clinical manifestation of alloimmune thrombocytopaenia or a complication of other diseases, such as intrauterine growth restriction, polycythaemia, sepsis or necrotising enterocolitis (NEC). ${ }^{3-58}$ Approximately $9.4 \%-35 \%$ of neonates admitted to neonatal intensive care units (NICUs) develop thrombocytopaenia. ${ }^{5-12}$ Theoretically, neonates with thrombocytopaenia may develop a high risk of bleeding and mortality. This increased risk is attributed to the important role of platelets in the whole process of haemostasis, and thrombocytopaenia may lead to dysfunctional haemostasis. Thus, this condition is a significant and unresolved problem for neonatologists.

Platelet transfusion (PT) is commonly used as a prophylactic and therapeutic treatment for bleeding episodes in neonates with 
thrombocytopaenia. To date, the relationship between a low platelet count and major bleeding or mortality is not clear, and the efficacy of PT remains controversial, as supported by the evidence from recent trials. ${ }^{5}{ }^{13-15}$ Current guidelines generally recommend prophylactic PT for neonates with thrombocytopaenia. ${ }^{16-19}$ The recommended thresholds vary from $20 \times 10^{9} / \mathrm{L}$ to $30^{15-1720-25} \times 10^{9} / \mathrm{L}$ for non-bleeding stable neonates, while the thresholds range from $30 \times 10^{9} / \mathrm{L}$ to $50^{152124-26} \times 10^{9} / \mathrm{L}$ for non-bleeding unstable neonates. These guidelines are consensus guidelines rather than evidence-based guidelines. ${ }^{197}$ Thus, a wide range of PT thresholds has been reported among different NICUs. ${ }^{28} 29$

Theoretically, compared with that at a low threshold, PT at a high threshold may reduce the risks of severe thrombocytopaenia, subsequent mortality and bleeding episodes. Surprisingly, a recent randomised controlled trial (RCT) reported that compared with PT at a low threshold, PT at a high threshold increased the mortality rate and bleeding events in preterm infants with severe thrombocytopaenia. ${ }^{14}$ On the other hand, as an invasive therapy, PT is associated with some acknowledged adverse events, including transfusion-transmitted infections, bacterial sepsis, febrile non-haemolytic transfusion reaction, transfusion-associated circulatory overload, transfusion-related acute lung injury and immunemediated platelet destruction. ${ }^{3}{ }^{30-32}$ Furthermore, PT has a higher risk of these adverse events than transfusions of other blood products due to its proinflammatory function.

Recently, additional clinical trials regarding PT in neonates with thrombocytopaenia have been completed. Several reports have argued that a lower transfusion threshold may reduce the incidence of unnecessary transfusions and financial costs without the extra risks of bleeding and mortality. ${ }^{13}$ We will perform this systematic review and meta-analysis to summarise current evidence for PT in neonates and assess the safety and best threshold for PT.

\section{Objectives}

We propose this protocol for a systematic review to collect and assess the evidence concerning the best threshold for PT to reduce mortality, bleeding and major morbidity among neonates with thrombocytopaenia. We will further explore the best thresholds for PT in neonates with thrombocytopaenia due to various causes and specific clinical characteristics. Furthermore, the safety of PT will be assessed by comparing its side effects at different thresholds.

\section{METHODS AND ANALYSIS}

This protocol will be conducted based on the Preferred Reporting Items for Systematic Review and Meta-Analysis Protocols 2015 guidelines, ${ }^{33}$ and a subsequent systematic review will be performed according to the Cochrane Handbook for Systematic Review of Interventions, ${ }^{34}$ the
Preferred Reporting Items for Systematic Reviews and Meta-Analyses (PRISMA) statement ${ }^{35}$ and the Grading of Recommendations Assessment, Development and Evaluation (GRADE) approach. ${ }^{36}$

\section{Data sources and search strategy}

Comprehensive searches will be separately performed by two independent researchers in the PubMed, Cochrane Library and Embase databases from database inception through 13 October 2020. No restrictions on language will be applied to the search. We will use the following keywords for the search and selection of relevant studies.

1. For neonates, the following combination of search terms will be used: "infant" or "newborn" or "neonatal" or "neonate" or "preterm" or "premature" or "neonatology".

2. For thrombocytopaenia, the following search terms will be used: "thrombocytopaenia" or "thrombocytopaenic" or "NT".

3. For PT, the following search terms will be used: "platelet transfusion" or "platelet infusion therapy" or "platelet administration" or "PT".

4. Steps 1, 2 and 3 will be combined with "and".

The detailed search strategy is shown in online supplemental table 1 .

Furthermore, we will manually check the references of all identified trials, relevant systematic reviews and current treatment guidelines to avoid missing important studies. Missing data will be handled by contacting relevant investigators for unreported materials or additional details.

\section{Study eligibility}

Types of studies

We will include RCTs, cohort studies and case-control studies and exclude animal studies, in vitro studies, crosssectional studies, case reports, case series and secondary or tertiary articles (systematic reviews and meta-analyses).

If enough data are available from only RCTs that will answer the questions posed by this review, we will report only data from RCTs.

\section{Types of participants}

Newborn infants with thrombocytopaenia (platelet counts $<150 \times 10^{9} / \mathrm{L}$, the diagnosis was established at less than 28 postnatal days, and the follow-up time could extend to a postnatal age $>28$ days) who were admitted to the NICU will be included. We will exclude studies of infants with congenital malformations. ${ }^{14}$

\section{Types of interventions and comparators}

The intervention of the included studies is PT for thrombocytopaenia. We will compare the effects of different transfusion platelet count thresholds and record the type and dose of the platelet component received.

\section{Types of outcomes}

The primary outcome will be in-hospital mortality or bleeding episodes (including intraventricular 
haemorrhage (IVH), intracranial haemorrhage, pulmonary haemorrhage, frank rectal bleeding and other bleeding).

The secondary outcomes will be morbidity (including patent ductus arteriosus, sepsis, NEC, bronchopulmonary dysplasia (BPD), retinopathy of prematurity (ROP), etc), adverse effects of transfusion and the length of stay (LOS). ${ }^{5} 142937-39$ Detailed descriptions of the outcome measures are provided in online supplemental table 2. If the data are sufficient, we will conduct additional analyses according to the severity of the outcomes (eg, severe IVH (grade III or IV)). The minimum length of follow-up for assessing these outcomes should include the time point for their diagnosis (eg, the follow-up for BPD should extend to 28 postnatal days). If a similar outcome measure had different follow-up times in different original studies, we will try to manage the data according to the timeline.

If the studies provide both adjusted and unadjusted results, only the adjusted results will be presented in the review.

\section{Study selection}

Two researchers will independently screen the titles and abstracts of the references retrieved by the searches. If eligible, the full texts of potential references will be obtained and assessed by the two researchers. Studies approved by both investigators will be included in this meta-analysis. Discrepancies in inclusion and exclusion decisions will be solved by a third senior researcher. Endnote X9 software will be used to track and manage the selection process, and a PRISMA flow diagram will be constructed to depict this process (see online supplemental figure 1).

\section{Data extraction}

Structured extraction sheets (see online supplemental tables 3.1-3.3) and Review Manager V.5.3 (Cochrane Collaboration, Oxford, UK) software will be used for data extraction by two independent investigators, and disagreements will be resolved by a third senior researcher. The included data items are as follows:

1. Publication and study details: authors, year of publication, country, study design and number of participants.

2. Clinical characteristics: gestational age (GA), birth weight (BW), platelet count before transfusion or severity of thrombocytopaenia, platelet count thresholds, type and dose of platelet component, and the number of PTs.

3. Outcomes: mortality, bleeding episodes, IVH grade, NEC, BPD, ROP, sepsis and LOS.

4. Other information: any sponsorship or funding.

Attempts will be made to retrieve missing information by contacting relevant investigators for unreported data or additional details.

\section{Risk of bias in individual studies}

Risk of bias will be assessed by two independent reviewers, and disagreement will be resolved by a third reviewer.

For RCTs, the 'Risk of Bias Assessment Tool' in Review Manager V.5.3 software (Cochrane Collaboration, UK) will be used. This tool includes random sequence generation (selection bias), allocation concealment (selection bias), blinding of participants and personnel (performance bias), blinding of outcome assessment (detection bias), incomplete outcome data (attrition bias), selective reporting (reporting bias) and other bias. The bias of the included studies will be divided into a high risk of bias, low risk of bias or unclear risk of bias in each domain (see online supplemental table 4$).{ }^{40}$

The Newcastle-Ottawa Scale will be used for observational studies in terms of selection, comparability and outcome, with a minimum score of 0 and a maximum score of 9 . Trials with scores of 9 points will be graded as high quality, and trials with scores of 1-8 points will be graded as low quality (see online supplemental table 5).

\section{Data synthesis}

When the studies are sufficiently homogeneous for any of the described outcome measures, a quantitative synthesis (meta-analysis) may be performed according to the recommendations of the Cochrane Handbook. If quantitative analysis cannot be performed, a narrative systematic review of the results from the studies included will be conducted, and we will not pool the data from the individual studies.

For dichotomous data (occurrence of mortality, bleeding events, morbidity, adverse events, etc), the risk ratio will be used in the analysis of RCTs and cohort studies and the OR will be used for case-control studies. For continuous data (GA, BW, etc), the mean difference (MD) or standardised MD with 95\% CIs will be used to represent the summary statistics of the outcome with the same units or different scales, respectively.

\section{Assessment of heterogeneity}

The $\chi^{2}$ test $(p \leq 0.1$ indicates substantial or considerable heterogeneity) will be used to determine whether heterogeneity is statistically significant. Additionally, we will assess the degree of statistical heterogeneity by examining $\mathrm{I}^{2}$. The data will be pooled by applying a random-effects model following $\mathrm{I}^{2} \geq 50 \%$ or $\mathrm{p} \leq 0.1$. Otherwise, the fixedeffects model will be used.

\section{Sensitivity analysis}

We will assess the robustness of the results by including or excluding controversial studies, such as low-quality studies or studies with temporal ambiguity (eg, whether the bleeding event occurred after PT is unknown).

\section{Subgroup analysis}

If sufficient data are identified, subgroup analyses will be performed to detect possible heterogeneity based on the following participant characteristics: 
1. GA ( $<28$ weeks, 28-32 weeks, 32-37 weeks and $>37$ weeks).

2. BW ( $<1000 \mathrm{~g}, 1000-1500 \mathrm{~g}, 1500-2500 \mathrm{~g}$ and $>2500 \mathrm{~g})$.

3. The severity of thrombocytopaenia (mild (100-150 $\left.\times 10^{9} / \mathrm{L}\right)$, moderate $\left(50-100 \times 10^{9} / \mathrm{L}\right)$ and severe $(<50$ $\left.\times 10^{9} / \mathrm{L}\right)$ ).

4. The platelet count thresholds for PT.

5. The cause of thrombocytopaenia.

6 . The design of the study (RCTs and cohort studies).

We will explore the possible heterogeneity among subgroups using $\mathrm{I}^{2}$ and $\mathrm{p}$ values.

\section{Quality of the evidence}

We will use the GRADE approach ${ }^{36} 40$ to assess the quality of evidence and propose to present 'Summary of findings' tables (see online supplemental table 6). We will construct funnel plots and perform the Egger's test to assess publication bias for each of the pooled outcomes when more than 10 included studies are available. Asymmetry may arise as a result of publication bias or a relationship between the trial size and effect size. Egger's linear regression analysis will be performed to test for funnel plot asymmetry.

\section{Patient and public involvement}

No patients will be involved.

\section{DISCUSSION}

Due to the limited number of RCTs, observational studies are a great source of potentially high-quality data. Furthermore, observational studies have additional benefits that may justify the evidence obtained from RCTs. We will include RCTs and observational studies in this review because of the limited number of relevant RCTs examining neonates with thrombocytopaenia. We will separately combine the results of RCTs and observational studies. To the best of our knowledge, this review will be the most recent systematic review determining the best PT threshold for neonates with thrombocytopaenia who are admitted to NICUs. We expect to provide the best available evidence for neonatologists and guideline developers on PT, which will help both clinical practice and further study design.

Contributors DL and JW are joint first authors. TX contributed to the conception of the study. The framework of the systematic review was developed by all authors. The search strategy was designed by TX and will be completed by YY and DL, who will further independently screen the relevant records, extract data from included studies and assess the risk of bias. JW will perform the data synthesis. TX and JT will arbitrate in cases of any disagreement and ensure that no errors occur during the study. The manuscript describing this protocol was drafted by DL and revised by TX. All authors have approved the publication of this protocol.

Funding This study is supported by grants from the Science and Technology Bureau of Sichuan Province (2020YJ0298 and 2020YJ0236); Health Commission of Sichuan Province (20PJ071); Deep Underground Space Medical Center (No. DUGM201809); and the Fundamental Research Funds for the Central Universities (SCU2019C4005).

Competing interests None declared.

Patient consent for publication Not required.
Provenance and peer review Not commissioned; externally peer reviewed.

Supplemental material This content has been supplied by the author(s). It has not been vetted by BMJ Publishing Group Limited (BMJ) and may not have been peer-reviewed. Any opinions or recommendations discussed are solely those of the author(s) and are not endorsed by BMJ. BMJ disclaims all liability and responsibility arising from any reliance placed on the content. Where the content includes any translated material, BMJ does not warrant the accuracy and reliability of the translations (including but not limited to local regulations, clinical guidelines, terminology, drug names and drug dosages), and is not responsible for any error and/or omissions arising from translation and adaptation or otherwise.

Open access This is an open access article distributed in accordance with the Creative Commons Attribution Non Commercial (CC BY-NC 4.0) license, which permits others to distribute, remix, adapt, build upon this work non-commercially, and license their derivative works on different terms, provided the original work is properly cited, appropriate credit is given, any changes made indicated, and the use is non-commercial. See: http://creativecommons.org/licenses/by-nc/4.0/.

\section{ORCID iDs}

Tao Xiong http://orcid.org/0000-0001-5255-6324

Jun Tang http://orcid.org/0000-0003-4884-4248

\section{REFERENCES}

1 von Lindern JS, van den Bruele T, Lopriore E, et al. Thrombocytopenia in neonates and the risk of intraventricular hemorrhage: a retrospective cohort study. BMC Pediatr 2011;11:16.

2 Roberts I, Stanworth S, Murray NA. Thrombocytopenia in the neonate. Blood Rev 2008;22:173-86.

3 Resch E, Hinkas O, Urlesberger B, et al. Neonatal thrombocytopeniacauses and outcomes following platelet transfusions. Eur J Pediatr 2018;177:1045-52

4 Ree IMC, Fustolo-Gunnink SF, Bekker V, et al. Thrombocytopenia in neonatal sepsis: incidence, severity and risk factors. PLoS One 2017;12:e0185581.

5 Dahmane Ayadi I, Ben Hamida E, Youssef A, et al. Prevalence and outcomes of thrombocytopenia in a neonatal intensive care unit. Tunis Med 2016;94:305-8.

6 Winkelhorst D, Oepkes D. Foetal and neonatal alloimmune thrombocytopenia. Best Pract Res Clin Obstet Gynaecol 2019;58:15-27.

7 Kamphuis MM, Paridaans NP, Porcelijn L, et al. Incidence and consequences of neonatal alloimmune thrombocytopenia: a systematic review. Pediatrics 2014;133:715-21.

8 Guida JD, Kunig AM, Leef KH, et al. Platelet count and sepsis in very low birth weight neonates: is there an organism-specific response? Pediatrics 2003;111:1411-5.

9 Bolat F, Kılıç Suar Çakı, Oflaz MB, et al. The prevalence and outcomes of thrombocytopenia in a neonatal intensive care unit: a three-year report. Pediatr Hematol Oncol 2012;29:710-20.

10 Castle V, Andrew M, Kelton J, et al. Frequency and mechanism of neonatal thrombocytopenia. J Pediatr 1986;108:749-55.

11 Mehta P, Vasa R, Neumann L, et al. Thrombocytopenia in the highrisk infant. J Pediatr 1980;97:791-4.

12 Ulusoy $\mathrm{E}$, Tüfekçi O, Duman N, et al. Thrombocytopenia in neonates: causes and outcomes. Ann Hematol 2013;92:961-7.

13 Andrew M, Vegh $\mathrm{P}$, Caco C, et al. A randomized, controlled trial of platelet transfusions in thrombocytopenic premature infants. $J$ Pediatr 1993;123:285-91.

14 Curley A, Stanworth SJ, Willoughby K, et al. Randomized trial of platelet-transfusion thresholds in neonates. $N$ Engl J Med 2019;380:242-51.

15 Borges JPG, dos Santos AMN, da Cunha DHF, et al. Restrictive guideline reduces platelet count thresholds for transfusions in very low birth weight preterm infants. Vox Sang 2013;104:207-13.

16 Girelli G, Antoncecchi S, Casadei AM, et al. Recommendations for transfusion therapy in neonatology. Blood Transfus 2015;13:484-97.

17 New HV, Berryman J, Bolton-Maggs PHB, et al. Guidelines on transfusion for fetuses, neonates and older children. $\mathrm{Br} J$ Haematol 2016;175:784-828.

18 Lopriore E. Updates in red blood cell and platelet transfusions in preterm neonates. Am J Perinatol 2019;36:S37-40.

19 Del Vecchio A, Franco C, Petrillo F, et al. Neonatal transfusion practice: when do neonates need red blood cells or platelets? Am J Perinatol 2016;33:1079-84.

20 Carr R, Kelly AM, Williamson LM. Neonatal thrombocytopenia and platelet transfusion - a UK perspective. Neonatology 2015;107:1-7. 
21 Calhoun DA, Christensen RD, Edstrom CS, et al. Consistent approaches to procedures and practices in neonatal hematology. Clin Perinatol 2000;27:733-53.

22 Sparger K, Deschmann E, Sola-Visner M. Platelet transfusions in the neonatal intensive care unit. Clin Perinatol 2015;42:613-23.

23 Christensen RD, Carroll PD, Josephson CD. Evidence-Based advances in transfusion practice in neonatal intensive care units. Neonatology 2014;106:245-53.

24 Baer VL, Lambert DK, Schmutz N, et al. Adherence to NICU transfusion guidelines: data from a multihospital healthcare system. $J$ Perinatol 2008;28:492-7.

25 Christensen RD, Paul DA, Sola-Visner MC, et al. Improving platelet transfusion practices in the neonatal intensive care unit. Transfusion 2008;48:2281-4.

26 Gibson BES, Todd A, Roberts I, et al. Transfusion guidelines for neonates and older children. Br J Haematol 2004;124:433-53.

27 Cremer M, Sallmon H, Kling PJ, et al. Thrombocytopenia and platelet transfusion in the neonate. Semin Fetal Neonatal Med 2016;21:10-18.

28 Fustolo-Gunnink SF, Huisman EJ, van der Bom JG, et al. Are thrombocytopenia and platelet transfusions associated with major bleeding in preterm neonates? A systematic review. Blood Rev 2019;36:1-9.

29 Kumar J, Dutta S, Sundaram V, et al. Platelet transfusion for PDA closure in preterm infants: a randomized controlled trial. Pediatrics 2019;143. doi:10.1542/peds.2018-2565. [Epub ahead of print: $02 \mathrm{Apr}$ 2019]

30 Haass KA, Sapiano MRP, Savinkina A, et al. Transfusion-Transmitted infections reported to the National healthcare safety network Hemovigilance module. Transfus Med Rev 2019;33:84-91.
31 Stolla M, Refaai MA, Heal JM, et al. Platelet transfusion - the new immunology of an old therapy. Front Immunol 2015;6:28.

32 Stainsby D, Jones H, Asher D, et al. Serious hazards of transfusion: a decade of hemovigilance in the UK. Transfus Med Rev 2006;20:273-82

33 Shamseer L, Moher D, Clarke M, et al. Preferred reporting items for systematic review and meta-analysis protocols (PRISMA-P) 2015 elaboration and explanation. BMJ 2015;350:g7647.

34 Higgins JPT, Thomas J, Chandler J, et al. Cochrane Handbook for systematic reviews of interventions. Type]: Chichester (UK: John Wiley \& Sons, 2019.

35 Moher D, Liberati A, Tetzlaff J, et al. Preferred reporting items for systematic reviews and meta-analyses: the PRISMA statement. Int $J$ Surg 2010;8:336-41.

36 Guyatt G, Oxman AD, Akl EA, et al. Grade guidelines: 1. IntroductionGRADE evidence profiles and summary of findings tables. J Clin Epidemiol 2011;64:383-94.

37 Roberts JC, Javed MJ, Hocker JR, et al. Risk factors associated with intraventricular hemorrhage in extremely premature neonates. Blood Coagul Fibrinolysis 2018;29:25-9.

38 Sancak S, Toptan HH, Gokmen Yildirim T, et al. Thrombocytopenia as a risk factor for retinopathy of prematurity. Retina 2019;39:706-11.

39 Jensen AK, Ying G-S, Huang J, et al. Longitudinal study of the association between thrombocytopenia and retinopathy of prematurity. J Aapos 2018;22:119-23.

40 Higgins JPT, Altman DG, Gøtzsche PC, et al. The Cochrane Collaboration's tool for assessing risk of bias in randomised trials 2011;343:d5928. 